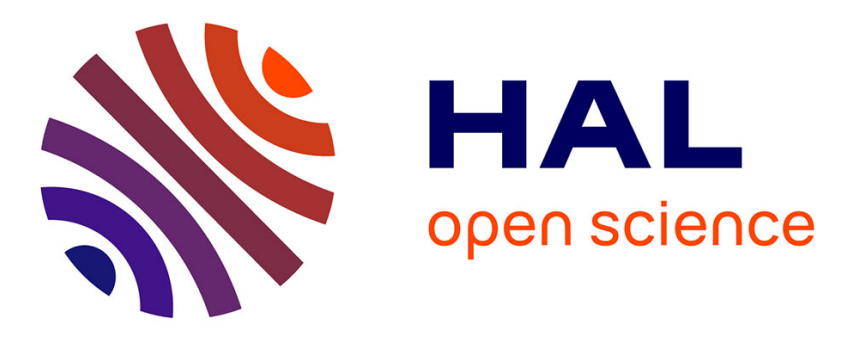

\title{
Unbiased Electro-Optic Waveguide as a Sensitive Nuclear Magnetic Resonance Sensor
}

Reina Aydé, Gwenaël Gaborit, Jean Dahdah, Lionel Duvillaret, Nadège

Courjal, Clément Guyot, Raphaël Sablong, Anne-Laure Perrier, Olivier Beuf

\section{- To cite this version:}

Reina Aydé, Gwenaël Gaborit, Jean Dahdah, Lionel Duvillaret, Nadège Courjal, et al.. Unbiased Electro-Optic Waveguide as a Sensitive Nuclear Magnetic Resonance Sensor. IEEE Photonics Technology Letters, 2014, 26 (12), pp.1266 - 1269. 10.1109/LPT.2014.2321099 . hal-01814491

\section{HAL Id: hal-01814491 \\ https://hal.univ-smb.fr/hal-01814491}

Submitted on 18 Jun 2018

HAL is a multi-disciplinary open access archive for the deposit and dissemination of scientific research documents, whether they are published or not. The documents may come from teaching and research institutions in France or abroad, or from public or private research centers.
L'archive ouverte pluridisciplinaire HAL, est destinée au dépôt et à la diffusion de documents scientifiques de niveau recherche, publiés ou non, émanant des établissements d'enseignement et de recherche français ou étrangers, des laboratoires publics ou privés. 


\title{
Unbiased Electro-Optic Waveguide as a Sensitive Nuclear Magnetic Resonance Sensor
}

\author{
Reina Aydé, Gwenaël Gaborit, Jean Dahdah, Lionel Duvillaret, Nadège Courjal, Clement Guyot, \\ Raphaël Sablong, Anne-Laure Perrier, and Olivier Beuf
}

\begin{abstract}
A pigtailed Ti: $\mathrm{LiNbO}_{3}$ waveguide is here associated to a specific nuclear magnetic resonant coil to perform a low invasive magnetic field measurement. The developed device exploits a passive electro-optic transduction between the measured magnetic field and polarization state modulation of a laser probe beam. Because of the use of integrated optics, the coil electromotive force induces a dramatically enhanced electric field, thus leading to sensitivity improvement. A minimum detectable magnetic field lower than $60 \mathrm{fT} \cdot \mathrm{Hz}^{-1 / 2}$ is achieved at the resonant frequency of $128 \mathrm{MHz}$. A dynamic range exceeding $100 \mathrm{~dB}$ is experimentally demonstrated.
\end{abstract}

Index Terms-Electro-optical devices, optical sensors, magnetic field measurement, nuclear magnetic resonance, magnetic resonance imaging.

\section{INTRODUCTION}

$\mathbf{M}$ AGNETIC Resonance Imaging (MRI) constitutes a non invasive technique which provides information related to the anatomy of a living being and allows to diagnosis certain diseases thanks to the analysis of soft tissues. Based on nuclear magnetic resonance (NMR), MRI consists in the study of magnetic modification properties of a nuclei which reflects its interaction with the environment (other nuclei and lattice) [1]. In order to extract this information, it is necessary to modify the magnetization from its equilibrium state, and then to detect its return to this equilibrium state driven by magnetic resonance frequency and different relaxations processes.

Manuscript received March 18, 2014; revised April 17, 2014; accepted April 28, 2014. Date of publication April 30, 2014; date of current version May 23, 2014. This work was supported in part by the Region Rhne-Alpes through the ADR and CIBLE Project, in part by the French Military Programs Management and Procurement Agency-Project RAPID SNIFER, in part by the Directorate General of Commercial Intelligence and Statistics, and in part by the LABEX PRIMES under Grant ANR-11-LABX-0063 through the Université de Lyon, within the Program Investissements d'Avenir under Grant ANR-11-IDEX-0007, operated by the French National Research Agency.

R. Aydé, R. Sablong, A.-L. Perrier, and O. Beuf are with CREATIS, Université de Lyon, Villeurbanne 69100, France (e-mail: reina.ayde@creatis.insa-lyon.fr; raphael.sablong@univ-lyon1.fr; anne-laure. perrier@creatis.insa-lyon.fr; anne-laure.perrier@creatis.insa-lyon.fr).

G. Gaborit is with the Laboratory of Microwave and Characterization, Université de Savoie, Le Bourget du Lac 73 376, France, and also with Kapteos, Le Bourget du Lac 73 376, France (e-mail: gwenael.gaborit@ univ-savoie.fr).

J. Dahdah and L. Duvillaret are with Kapteos, Le Bourget du Lac 73 376, France (e-mail: jean.dahdah@kapteos.com; lionel.duvillaret@kapteos.com).

N. Courjal and C. Guyot are with the Institut Femto-St, Universit de Franche-Comt, Besanon 25030, France (e-mail: nbodin@univ-fcomte.fr; clement.guyot@femto-st.fr).
Conventionally, an inductive loop is used to characterize the magnetization variations via the induced electromotive force (EMF). The measurement is usually performed with an external coil to image the biological media [2]. However, despite technical MRI improvements, spatial resolution and achievable image quality with external coil are still limited for the examination of deep regions. It has been demonstrated that an endoluminal (internal) coil provides an important increase in local signal to noise ratio (SNR), and enables very high resolution images [3]. Nevertheless, the use of metallic coaxial cable, connecting the coil to the MR system could induce a considerable local specific absorption rate (SAR) especially around this cable [4], [5]. In fact, during a MRI experiment, the radiofrequency (RF) magnetic field is accompanied by an electric (E) field, thus inducing current flow in the metallic cable at the same frequency called common mode. Number of solutions were proposed to reduce this current induced heating [7]-[9]. However, these solutions were not efficient all along the coaxial cable. Optical fibre link can be used to overcome heating problems and to ensure patients safety. In general, to transmit NMR signal from coil to MR system, the direct modulation of laser diode intensity fixed on the probe is applied [10]-[12]. Since direct current supply is required, these methods remain invasive.

Magnetic field characterization involving passive optical system has been already performed [13], [14]. This type of magnetic probe is based on an electro-optic (EO) material associated to a magnetic loop inducing an electric (E)-field proportional to a magnetic field component. This experimental configuration allows to minimize the invasiveness: the probe can be pigtailed, does not need power supply and includes metallic element much shorter than the wavelength of the field to be measured. This kind of sensor has been studied by Suzuki et al. [15] and exploits a magnetic loop doubly loaded with $\mathrm{LiNbO}_{3}$ crystal, acting as the capacitance of the resonator. The realized set-up is dedicated to measure the magnetic field propagating along a stripline, over a wideband of frequencies in the microwave range. The goal is here to develop a resonant EO probe dedicated to RF magnetic field measurement. As our field of interest concerns MRI, the resonant frequency is precisely defined (e.g. $128 \mathrm{MHz}$ corresponding to the proton frequency at $3 \mathrm{~T}$ ). Moreover, this resonant frequency is lower in MRI system compared to the microwave frequencies, thus inducing a lower electromotive force $\epsilon$ applied to the crystal $\left(\epsilon=-\frac{d \phi}{d t}\right.$ with $\phi$ the magnetic flux). A setup involving a bulk millimetre-sized $\mathrm{LiTaO}_{3}$ crystal 
has been already analysed [16]. The results were in a very good agreement with the theoretical expectations. But using a thinner crystal will lead to a higher internal electric field, thus to an enhanced sensitivity. Moreover, a smaller sensor induces lower disturbances on the external field to be measured. Hence, the purpose of the study is to develop an equivalent set up associated to a matched resonant loop dedicated to magnetic field measurements, where a Ti: $\mathrm{LiNbO}_{3}$ waveguide replaces a bulk crystal. In the first section, the waveguide transducer performs the E-field induced polarization state modulation (PSM) of a laser beam, and its intrinsic E-field sensitivity is analysed. Subsequently, the EO chip is coupled to the resonant coil to perform magnetic field measurement. The device is optimized in terms of impedance matching. Its linearity response with respect to the magnetic field magnitude is then analysed. The sensitivity is compared to the one obtained with a bulk crystal instead of the optical EO waveguide. The result demonstrates the sensitivity improvement.

\section{EO TRANSDUCtion USING AN INTEGRATED $\mathrm{LiNbO}_{3}$ WAVEGUIDE}

The Pockels effect acts in non-centrosymetric crystals. This EO effect traduces the linear link between the field $\vec{E}$ to be measured and the induced modification of index ellipsoid of the crystal. A detailed study of EO crystal can be found in Ref. [17] and [18]. The developed transducer is based on a $\mathrm{LiNbO}_{3}$ crystal. The corresponding EO tensor which gives the vectorial dependence of index ellipsoid with the applied electric field $\vec{E}$ is given below:

$$
\left(\begin{array}{lll}
0 & -r_{22} & r_{13} \\
0 & r_{22} & r_{13} \\
0 & 0 & r_{33} \\
0 & r_{51} & 0 \\
r_{51} & 0 & 0 \\
-r_{22} & 0 & 0
\end{array}\right)
$$

This tensors involves the EO coefficients $r_{i j}$ providing the vectorial behaviour of the $\mathrm{EO}$ measurement, i.e. the dependence of the refractive indices $n_{i}$ to the components $E_{j}$ of the field vector:

$$
\delta\left(\frac{1}{n^{2}}\right)_{i}=\sum_{j=1}^{3} r_{i j} E_{j}
$$

The optical wave crossing the crystal sees actually two eigen indices $n_{+}$and $n_{-}$. As we here exploit the E-field induced modification of the polarization state of a laser beam, the relevant information is $\delta n(\vec{E})=\delta\left(n_{+}(\vec{E})-n_{-}(\vec{E})\right.$. The E-field induced modifications of the eigen indices are much lower than the intrinsic indices and this latter equation can be rewritten as follow [18]:

$$
\delta n(\vec{E})=\overrightarrow{\Delta K} \cdot \vec{E}
$$

The differential sensitivity vector $\overrightarrow{\Delta K}$ gives the direction of electric field component probed by the EO crystal and its modulus leads to the sensitivity of the EO conversion. $\overrightarrow{\Delta K}$ is given by:

$$
\overrightarrow{\Delta K}=\left.\vec{\nabla} \delta n\left(\overrightarrow{E_{\Omega}}\right)\right|_{\overrightarrow{E_{\Omega}}=0}
$$

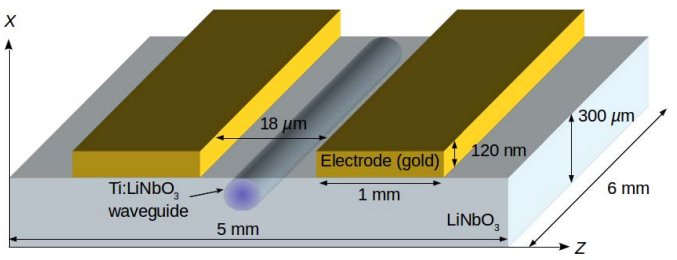

Fig. 1. Schematic of the $\mathrm{LiNbO}_{3}$ chip ensuring the EO transduction.

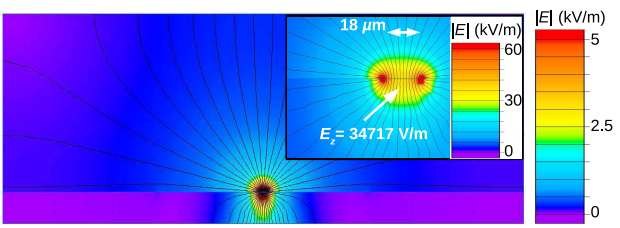

Fig. 2. Transverse FDTD simulations of the field induced by the electrode potentials and surrounding the $\mathrm{LiNbO}_{3}$ chip. A potential difference of $1 \mathrm{~V}$ is applied on the electrodes. Equipotential lines are indicated in black and field strength is linked to the color, from purple to red. The inset corresponds to a zoom on the location of the optical probe beam, in between the electrodes. The respective field scales are given on the left side of each image.

$\overrightarrow{\Delta K}$ depends only on EO crystal properties and on the probe beam direction $\vec{k}$ relatively to the crystal axis. Considering an $x$-cut wafer of $\mathrm{LiNbO}_{3}$ with the optical wave propagating along the $y$-axis of the crystal (see Fig. 1), the sensitivity to the field component $E_{z}$ takes the value $\left|\overrightarrow{\Delta K}_{y-p r o p, E_{z}}\right|$. As $r_{i j} \ll n^{-2}$, the modulus of this sensitivity vector becomes:

$$
\left|\overrightarrow{\Delta K}_{y-p r o p, E_{z}}\right|=\left|\frac{1}{2}\left(n_{z}{ }^{3} r_{33}-n_{x}{ }^{3} r_{13}\right)\right|
$$

with $n_{x}=n_{+}=2.20$ and $n_{z}=n_{-}=2.13$, the eigen indices seen by the optical wave, $r_{13}=8.6 \mathrm{pm} / \mathrm{V}$ and $r_{33}=30.8 \mathrm{pm} / \mathrm{V}$, the EO coefficients involved in the $E_{z} \mathrm{com}$ ponent probing. The field $\left|E_{z}\right|$ to be probed is induced by the EMF $\epsilon=V_{z}$, corresponding to the potential difference applied to the gold electrodes. Hence, the separating distance $d$, between the electrodes is here of major importance in order to reach very high sensitivity: while electrodes placed on sides of a bulk crystal are usually separated by a distance lying in the millimetre range, the optical waveguide transverse dimensions are approximately $10 \mu \mathrm{m}$ (for the optical wavelength $\lambda_{\text {opt }}=1550 \mathrm{~nm}$ ), leading potentially to a significant enhancement of the induced field $\left(E_{z} \propto \frac{V_{z}}{d}\right)$, so that to the response of the sensor.

The transverse transducer consists in a Ti-diffused $\mathrm{LiNbO}_{3}$ waveguide (length $L_{y}=6 \mathrm{~mm}$, in agreement with the NMR coil dimensions) confined in between two coplanar electrodes lying in the $y z$ plane and separated by a distance $d=18 \mu \mathrm{m}$. This distance ensures a sensitivity enhancement together with an homogeneous E-field (in modulus and direction) seen by the optical beam. Moreover this configuration induces a capacitance which can be compensated (see section III). A schematic of the EO chip is proposed in Fig.1. A FDTD simulation (Quickfield ${ }^{\circledR}$ ) has been performed in order to assess the induced electric field $\vec{E}_{z}$ between electrodes, in the optical waveguide location. The result is given is in Fig.2.

This E-field $E_{z}$ will lead to a dephasing between the two allowed polarization state inside the EO crystal and is finally 


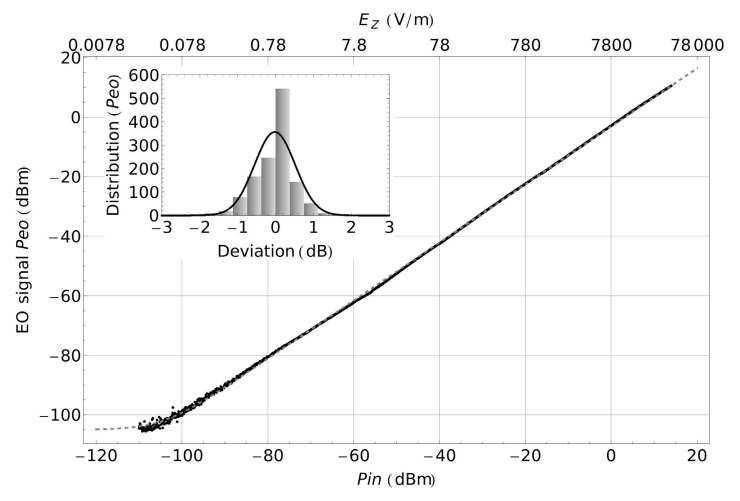

Fig. 3. EO response as a function of the frequency synthesizer delivered power $(f=128.2 \mathrm{MHz}, R B W=30 \mathrm{~Hz})$. Measurement data and theoretical fit are linked to black dots and gray dashed line respectively. The inset corresponds to the deviation between measurement data and theoretical fitting curve.

written:

$$
\Delta \varphi=\frac{2 \pi}{\lambda_{o p t}}\left|\overrightarrow{\Delta K}_{y-p r o p, E_{z}}\right| \cdot E_{z} L_{y}
$$

The PSM is finally optically treated with a quarter wave plate to compensate the static birefringence of the crystal, and with a polarizer to convert the PSM into a modulation of optical power. An amplified high speed photodiode ensures the last conversion to an electrical signal.

The response of the realized waveguide EO transducer has been characterized. For that purpose, a synthesizer feeds the electrodes at the frequency of interest $(f=128 \mathrm{MHz})$ and the EO signal $P_{E O,[\mathrm{dBm}]}$ is recorded with a spectrum analyser as a function of the delivered power $P_{i n,[\mathrm{dBm}]}$. Fig.3 illustrates the obtained result. The experimental characterization can be fitted with the equation involving the delivered power itself as well as the noise contribution:

$$
P_{E O[d B m]}=10 \log _{10}\left(10^{\frac{\text { Noise }_{[\mathrm{dBm}]}}{10}}+10^{\frac{\alpha P_{i n,[\mathrm{dBm}]}+\beta}{10}}\right)
$$

The coefficients $\alpha$ and $\beta$ are the linearity factor and the conversion efficiency, respectively. This latter one is determined by the EO conversion itself $(\Delta \varphi)$, but also to the optical power carrying the modulation $(1 \mathrm{~mW})$ and to the photodiode responsivity $(0.85 \mathrm{~A} / \mathrm{W})$.

The measurements dynamic range exceeds $100 \mathrm{~dB}$. The minimum detectable field $E_{\min }$ is obtained equalizing the linear behaviour of the chip and the Johnson-Nyquist noise floor of the spectrum analyser and of the photodiode electronics $($ Noise $[\mathrm{dBm}]=-105 \mathrm{dBm}) . E_{\min }$ is here lower than $10 \mathrm{mV} \cdot \mathrm{m}^{-1} \cdot \mathrm{Hz}^{-1 / 2}$. This latter value corresponds to a root mean square (RMS) voltage $V_{z}<0.3 \mu \mathrm{V}$. Moreover the linear slope of the measurement is $\alpha=0.98$ and the standard deviation of the error distribution is about $0.5 \mathrm{~dB}$ (see inset of Fig.3). The conversion efficiency between $P_{i n}$ and $P_{E O}$ is $\beta=-3 \mathrm{~dB}$.

\section{The Electro-Optic Resonant CoIL}

Based on previous work [19], a reference rectangular coil was built with an external width set to $5.1 \mathrm{~mm}$ and a length

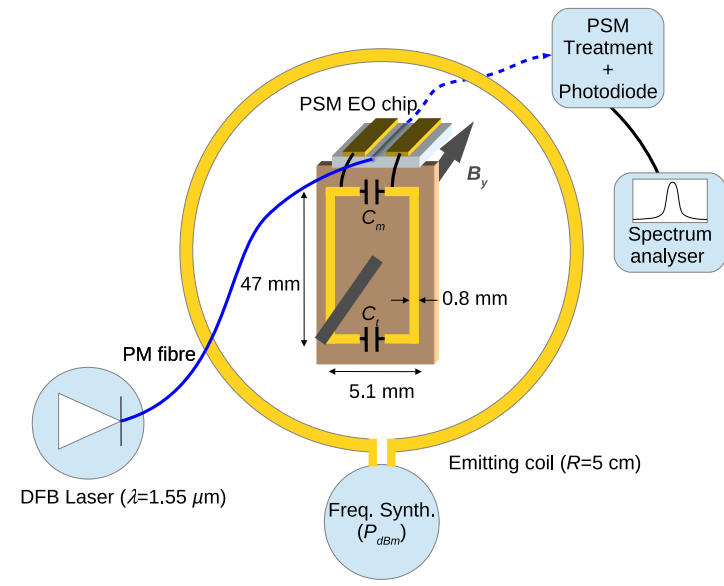

Fig. 4. Implementation scheme of the $\mathrm{EO} \mathrm{LiNbO}_{3}$ waveguide and experimental setup dedicated to the magnetic field response analysis. DFB Laser: distributed feeback Laser; PM fibre: polarization maintaining fibre.

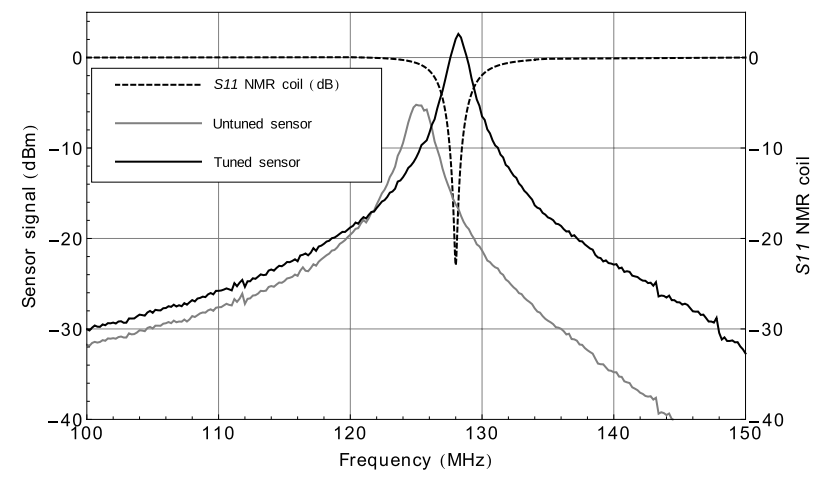

Fig. 5. Frequency response of the EO coil $\left(P_{i n}=14 \mathrm{dBm}, R B W=30 \mathrm{~Hz}\right)$. The black dashed line corresponds to the return loss $S 11$ of the initially designed coil itself. Gray curve and black curves correspond to the unmatched sensor and to the $\mathrm{LiNbO}_{3}$ chip matched coil, respectively. An increase of $19.5 \mathrm{~dB}$ is obtained at the nominal frequency of $128 \mathrm{MHz}$.

set to $47 \mathrm{~mm}$. The coil is constituted with $35 \mu \mathrm{m}$ thickness copper tapes on a substrate FR4 (relative permittivity $\epsilon_{r}=4$ and thickness $T=0.8 \mathrm{~mm}$ ). These size and geometry provide good image with adequate penetration depth and exploration length. Moreover, the dimensions are in agreement with further developments dedicated to endoluminal sensor. The coil is matched to $50 \Omega$ at a frequency of $128 \mathrm{MHz}$ thanks to matching and tuning nonmagnetic capacitors on distal and proximal part of the coil. The initial values of these capacitors are $C_{t}=36.2 \mathrm{pF}$ and $C_{m}=186.8 \mathrm{pF}$ for tuning and matching respectively. The resonance of the coil itself has been firstly analysed via the measurement of its $S 11$ parameter. The design actually leads to a minimum return loss at the expected frequency (see dashed curve on Fig.5). In order to realize a magnetic sensor, the coil is connected to the EO chip via bonding wires. The experimental setup dedicated to characterize the magnetic sensor is prensented in Fig. 4. A RF synthesizer feeds an emitting coil, providing a $128 \mathrm{MHz}$ RF magnetic field $B_{y}$. The endoluminal coil converts the $B_{y}$ field into a RF E-field. The EO signal $P_{E O}$ is recorded with a spectrum analyser. The measurement obtained with the sensor is presented in Fig.5 


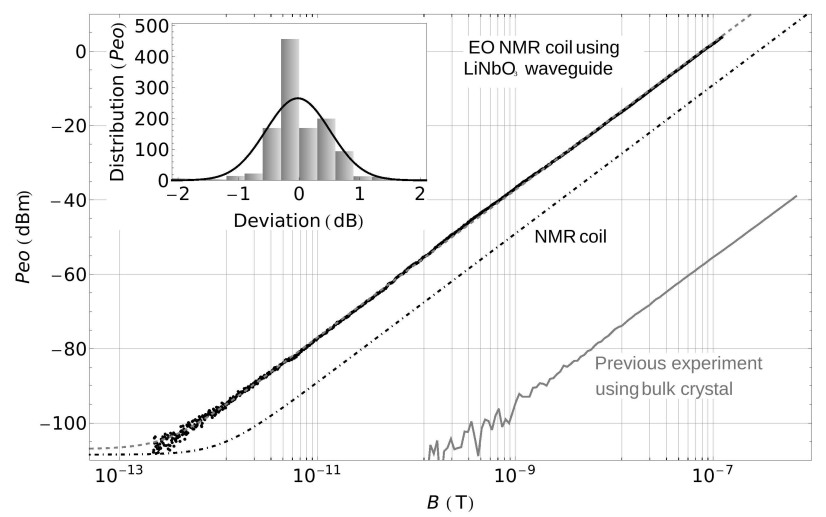

Fig. 6. EO NMR coil response as a function of the applied magnetic field. ( $f=128 \mathrm{MHz}, R B W=30 \mathrm{~Hz}$ ). Black dots and gray dashed line corresponds to measurement data and fitting curve respectively. Inset illustrates to the deviation between measurement data and theoretical fitting curve. Additional gray curve is linked to a previous measurement obtained with a bulk $\mathrm{LiTaO}_{3}$ crystal $4 \mathrm{~mm}$-thick. Dot-dashed line indicates the intrinsic resonant coil (galvanic linked) response.

(gray line). The curve shows that the addition of the EO chip shifts the sensor resonance toward lower frequencies, due to its intrinsic and non-negligible capacitance. In order to retrieve the nominal resonance, $C_{t}$ has been adjusted to $35.2 \mathrm{pF}$ to compensate capacitance value of the EO chip and inductance of bonding connection. Measurement of $P_{E O}$ in this case is presented with the black line of Fig. 5.

The linearity response of the realized EO coil has been investigated as a function of the applied magnetic field. The result is presented in Fig. 6. Once again, the linearity measurement exhibits a dynamic range greater than $100 \mathrm{~dB}$. The fitting curve gives the magnetic field $B$ response and is written:

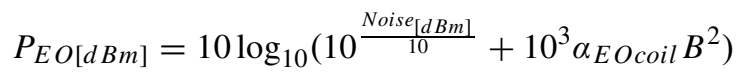

The coefficient $\alpha_{E O \text { ocoil }}$ is the link between the EO resonant coil signal and the magnetic field to be probed. $\alpha_{E O \text { ocoil }}$ takes here a value of $1.27 \times 10^{11} \mathrm{~W}^{\mathrm{T}}{ }^{-2}$, which corresponds to a linear coefficient of $\sqrt{R \alpha_{\text {EOcoil }}}=2.5 \times 10^{6} \mathrm{~V}^{\mathrm{T}} \mathrm{T}^{-1}$, $R=50 \Omega$ being the resistive load. This measurement allows to determine the minimum detectable magnetic field $B_{\min }=0.3 \mathrm{pT}$ in the $30 \mathrm{~Hz}$ resolution bandwidth (RBW) leading to $B_{\min }=56 \mathrm{fT} \cdot \mathrm{Hz}^{-1 / 2}$. Furthermore, this measurement is compared to a similar setup involving a bulk $\mathrm{LiTaO}_{3}$ crystal (4 mm thick) [16]. An increase in sensitivity is demonstrated and reaches more than $57 \mathrm{~dB}$, without any additional amplification of the signal. Finally, the equation 8 can be applied for the magnetic field response of the intrinsic NMR coil in its nominal use (galvanic linked and $50 \Omega$ loaded, see dotdashed line on Fig. 6). It allows to determine $\alpha_{\text {Coil }}=1.23 \times 10^{10} \mathrm{~W} \mathrm{~T}^{-2}$, ten times lower than the achieved value of $\alpha_{E O \text { coil }}$ (with the fibre link and the EO conversion).

\section{CONCLUSION}

An EO waveguide has been coupled to a resonant coil to design a pigtailed deported and sensitive magnetic field sensor. The use of integrated optics allows to apply the coil EMF on a weak separating distance leading to the enhancement of the field strength seen by the laser probe beam. The passive EO chip exhibits a linear response over a dynamic range exceeding $100 \mathrm{~dB}$. A magnetic field as low as $300 \mathrm{fT}$ remains detectable at the resonant frequency $f=128 \mathrm{MHz}$. The sensor response is increased by more than $50 \mathrm{~dB}$ compared to the use of a bulk crystal and by $10 \mathrm{~dB}$ compared to the galvanic linked NMR coil. Finally, the millimetre size and the composition of the device are fully suitable for further developments concerning low-invasive and endoluminal MR coils.

\section{REFERENCES}

[1] B. Kastler, Understanding MRI. Berlin, Germany: Springer-Verlag, 2008.

[2] J. T. Vaughan and J. R. Griffiths, RF Coils for MRI. Hoboken, NJ, USA: Wiley, 2012.

[3] O. Beuf, F. Pilleul, M. Armenean, G. Hadour, and H. Saint-Jalmes, "In vivo colon wall imaging using endoluminal coils: Feasibility study on rabbits," J. Magn. Reson. Imag., vol. 20, no. 1, pp. 90-96, 2004.

[4] C. J. Yeung, R. C. Susil, and E. Atalar, "RF safety of wires in interventional MRI: Using a safety index," in Proc. 23rd Annu. Int. Conf. IEEE Eng. Med. and Biol. Soc., Jan. 2001, pp. 2496-2498.

[5] C. Armenean, E. Perrin, M. Armenean, O. Beuf, F. Pilleul, and H. Saint-Jalmes, "RF-induced temperature elevation along metallic wires in clinical magnetic resonance imaging: Influence of diameter and length," Magn. Reson. Med., vol. 52, no. 5, pp. 1200-1206, 2004.

[6] V. Detti, D. Grenier, E. Perrin, and O. Beuf, "Assessment of radiofrequency self-heating around a metallic wire with MR T1-based thermometry," Magn. Reson. Med., vol. 66, no. 2, pp. 448-455, 2011.

[7] M. Ladd and H. Quick, "Reduction of resonant RF heating in intravascular catheters using coaxial chokes," Magn. Reson. Med., vol. 43, no. 4, pp. 615-619, 2000.

[8] S. Weiss, P. Vernickel, T. Schaeffter, V. Schulz, and B. Gleich, "Transmission line for improved RF safety of interventional devices," Magn. Reson. Med., vol. 54, no. 1, pp. 182-189, 2005.

[9] D. Peterson, B. Beck, G. R. Duensing, and J. Fitizsimmons, "Common mode signal rejection methods for MRI: Reduction of cable shield currents for high static magnetic field systems," Concepts Magn. Reson. B, Magn. Reson. Eng., vol. 19, no. 1, pp. 1-8, 2003.

[10] C. Du, J. Yuan, and G. X. Shen, "Comparison of FP, VCEL and DFB laser diode in optical transmission for MR RF coil array," in Proc. 15th Annu. Conf. ISMRM, vol. 15. 2007, p. 1041.

[11] J. Yuan, J. Wei, and G. X. Shen, "A 4-channel coil array interconnection by analog direct modulation optical link for 1.5-T MRI," IEEE Trans. Med. Imag., vol. 27, no. 10, pp. 1432-1438, Oct. 2008.

[12] O. G. Memis, Y. Eryaman, O. Aytur, and E. Atalar, "Miniaturized fiberoptic transmission system for MRI signals," Magn. Reson. Med., vol. 59, no. 1, pp. 165-173, 2008.

[13] E. Suzuki, S. Arakawa, H. Ota, K. I. Arai, and R. Sato, "Integrated optics magnetic sensor from $2 \mathrm{kHz}$ to $9 \mathrm{GHz}, "$ Appl. Opt., vol. 50, no. 11, pp. 1570-1574, 2011.

[14] A. Mhapatra and V. E. Stenger, "Electro-optic electromagnetic field sensor system with optical bias adjustment," U.S. Patent, 5963034, Oct. 5, 1999.

[15] E. Suzuki, S. Arakawa, H. Ota, K. I. Arai, and R. Sato, "Optical magnetic field probe with a loop antenna element doubly loaded with electrooptic crystals," IEEE Trans. Electromagn. Compat., vol. 46, no. 4, pp. 641-647, Nov. 2004.

[16] R. Aydé et al., "Potentialities of an electro-optic crystal fed by nuclear magnetic resonant coil for remote and low-invasive magnetic field characterization," IEEE Sensors J., vol. 13, no. 4, pp. 1274-1280, Apr. 2013.

[17] A. Yariv, Optical Electronics. New York, NY, USA: Saunders, 1991.

[18] L. Duvillaret, S. Rialland, and J.-L. Coutaz, "Electro-optic sensors for electric field measurements. II. Choice of the crystals and complete optimization of their orientation," J. Opt. Soc. Amer. B, vol. 19, no. 11, pp. 2704-2715, 2002.

[19] M. Armenean, O. Beuf, F. Pilleul, and H. Saint-Jalmes, "Optimization of endoluminal loop radiofrequency coils for gastrointestinal wall MR imaging," IEEE Sensors J., vol. 4, no. 1, pp. 57-64, Feb. 2004. 